\title{
Urgences
}

\section{L'Amérique et l'américanité chez Jacques Poulin}

\section{Anne Marie Miraglia}

Numéro 34, décembre 1991

Mythes et Romans de l'Amérique

URI : https://id.erudit.org/iderudit/025683ar

DOI : https://doi.org/10.7202/025683ar

Aller au sommaire du numéro

Éditeur(s)

Urgences

ISSN

0226-9554 (imprimé)

1927-3924 (numérique)

Découvrir la revue

Citer cet article

Miraglia, A. M. (1991). L'Amérique et l'américanité chez Jacques Poulin.

Urgences, (34), 34-45. https://doi.org/10.7202/025683ar d'utilisation que vous pouvez consulter en ligne.

https://apropos.erudit.org/fr/usagers/politique-dutilisation/ 


\section{L'Amérique et l'américanité chez Jacques Poulin}

\section{Anne Marie Miraglia}

Quelle que soit la définition que l'on attribue au terme, il est clair que l'*américanité * de l'écriture poulinienne se manifeste sur differents plans de l'œuvre - linguistique, stylistique, thématique aussi bien qu'intertextuel. Il s'agit donc d'une question complexe qui devrait amener une multiplicité d'approches différentes mais qui jusqu'à présent s'est limitée à l'énumération de références à l'écriture américaine (surtout à l'œuvre d'Ernest Hemingway) et à d'autres produits culturels américains - films, chansons, marchandises, etc. Or la critique n'a pas encore essayé de trancher entre ce qui chez Poulin relève d'«influences * et ce qui relève d'* affinités * avec la littérature américaine. Autrement dit, elle n'a pas encore différencié l'" américanisation " de l'écriture poulinienne de son *américanité". Ces deux termes sont à distinguer: le premier exprime ici un mode de vie - celui de la consommation à l'américaine (états-unienne) ainsi que ses conséquences sur la langue, la littérature et la culture québécoise; le deuxième, par contre, signale un état d'esprit, c'est-à-dire la conscience d'appartenir au continent nord-américain, de jouer un rôle dans son Histoire et de faire partie d'une nouvelle race humaine. La pénétration progressive de la culture américaine dans les romans de Poulin coïncide avec l'ouverture de l'espace fictif poulinien et, en général, avec la disponibilité du Québec contemporain à explorer son américanité.

L'américanisation de l'écriture poulinienne se manifeste le plus clairement dans l'accumulation d'allusions reflétant l'assimilation d'une culture made in the U.S.A. Il en est ainsi, par exemple, des bandes dessinées américaines qui sont traduites par Teddy dans Les grandes marées et qui font l'objet principal des recherches de Mocassin, professeur à la Sorbonne. Il faut voir dans la traduction même de ces bandes dessinées un signe de l'emprise de la culture populaire américaine au Québec comme en France. Mais il faudrait aussi reconnaître que si, dans Les grandes marées, le discours 
littéraire états-unien est simplement décodé et reproduit en traduction, il est, par contre, dans Volkswagen Blues, générateur d'une œuvre originale et essentiellement québécoise. Dans cette dernière, il est toutefois difficile de distinguer entre l'américanisation et l'américanité de l'écriture poulinienne. L'œuvre littéraire du romancier fictif Jack Waterman y est assez souvent juxtaposée à celle des écrivains américains John Irving, Saul Bellow et, en particulier, à celle de Jack Kerouac. L'écriture de Volkswagen Blues semble se faire à partir de la lecture (donc la consommation) de romans américains portant sur les mêmes thèmes, en l'occurrence l'Amérique et le rêve américain. Si les thèmes dans ce roman de Poulin font écho aux préoccupations principales de ses intertextes americains (The Hotel New Hampshire, The Adventures of Augie March, On the Road, The Valley of the Moon, etc.), faut-il conclure qu'il est question d'火influences * ou s'agit-il plutôt de l'expression d'une écriture ancrée profondément dans le tellurisme nord-américain? Examinons brièvement, à titre d'illustration, le passage suivant:

[...] ils comprirent tous les deux et sans avoir besoin de se dire un mot que c'était le Mississippi, le Père des Eaux, le fleuve qui séparait l'Amérique en deux et qui reliait le Nord et le Sud, le grand fleuve de Louis Jolliet et du père Marquette, le fleuve sacré des Indiens, le fleuve des esclaves noirs et du coton, le fleuve de Mark Twain et de Faulkner, du jazz et des bayous, le fleuve mythique et légendaire dont on disait qu'il se confondait avec l'âme de l'Amérique. ${ }^{1}$

Il est clair ici que, même si Jack Waterman et Pitsémine ne sont pas Américains (ici au sens d'"États-Uniens»), le signe "Mississippi " suscite chez eux une série d'associations et de lieux communs identiques. Dans ce cas, l'écrivain québécois et la Métisse partagent, de toute évidence, un code basé essentiellement sur des expériences littéraires et culturelles nord-américaines. Mais comment différencier nettement les éléments qui relèvent de leur américanisation de ceux qui relèvent de leur américanité? Cette question pourrait se poser aussi à l'égard de l'auteur lui-même.

1 Jacques Poulin, Volkswagen blues, Montréal, Québec/Amérique, 1984, p. 117-118. 
36

Je ne me propose pas de traiter la problématique américanisation / américanité et ses diverses manifestations textuelles, sujet évidemment trop vaste pour le cadre de cette analyse, je me propose, par contre, d'étudier un seul aspect de cette problématique en me penchant sur la question identitaire thématisée dans Volkswagen Blues. Je montrerai que le discours identitaire s'y exprime par l'intermédiaire de la figure du frère Théo et est coloré par les relations binaires douceur/agressivité, sédentarité/nomadisme, francité/américanité $\hat{~}^{2}$. Le questionnement identitaire, cependant, n'est pas particulier à Volkswagen Blues; il se retrouve dans tous les romans de Poulin et surtout dans ceux où un personnage scripteur (écrivain, commis aux écritures, etc.), mal à l'aise dans ses rapports avec autrui, cherche par le biais de l'écriture à mieux se connaître et à mieux se faire comprendre.

La question identitaire comporte toujours chez Poulin une dimension nationale et se pose pour la première fois explicitement dans Le cceur de la baleine bleue où Noël, le romancier fictif, reconnaît tristement ne pas se sentir «Américain»: «Pas dans le sens de connaître Tchaikowsky [sic] par Walt Disney. Plutôt dans le sens d'avoir au cœur l'espoir fou qu'on peut tout faire de force. Je ne me sens pas citoyen de l'Amérique [...] Il n'y a plus de place pour la douceur. " ${ }^{3}$ Dans cette définition d' "Américain *, Noël semble vouloir opposer l'acquisition d'une culture états-unienne (donc américanisation) au sentiment d'appartenance physique et spirituelle au continent nord-américain (ou américanité). Or l'aliénation vis-à-vis de l'Amérique dont parle Noël ne lui est pas spécifique mais témoigne, en général, d'un état d'esprit essentiellement commun aux Québécois. Voici ce qu'en dit Gilles Marcotte:

Quand je pense Amérique, je pense d'abord États-Unis d'Amérique [...] puis viendra l'Amérique du Sud; enfin je penserai peut-être au Canada, au Québec, mais avec

2 Cette dernière relation binaire composant l'identité québécoise est au centre des numéros d'Études françaises vol. $X, n^{\circ} 4$, novembre 1974, d'Études littéraires, vol. VIII, $n^{\circ} 1$, avril 1975, de Liberté 138, novembre-décembre 1981 et d'Etudes françaises vol. XVI, n`2, automne 1990.

3 Jacques Poulin, Le cceur de la baleine bleue, Montréal, Les Editions du Jour, 1979, p. 62. 
certaines réticences, un certain malaise car dans mon expérience de parole et de lecture le mot Amérique ne se superpose pas. II évoque pour mol, d'entrée de jeu, une réalité étrangère - presque autant que la vieille Europe. 4

Mais des Québécois comme, par exemple, Jean Morisset ${ }^{5}$ revendiquent de plus en plus une identité fondée sur leur appartenance et leur participation historique et culturelle au continent nord-américain, ce que semble dénier l'appellation plutôt insulaire de * Québécois *. Pour ces critiques, l'identité québécoise se fonde moins sur ses liens avec la France et davantage sur ses rapports avec l'Amérique et forcément avec les États-Unis.

La France et les États-Unis - voilà les deux pôles d'attraction qui continuent à tirailler le Québec et à nourrir l'identité québécoise. Il semble toutefois que, dans les romans de Poulin, le salut identitaire se trouve justement dans cet entre-deux. Ainsi dans Les grandes marées, l'Auteur n'accepte de s'identifier exclusivement ni au Français, ni à l'Américain ${ }^{6}$. Dans Volkswagen Blues, l'écrivain Jack Waterman ne se considère "pas tout à fait * français : *On est Québécois * (p. 270). Dans ce roman, être * québécois * ou la québécitude semble impliquer la fusion à la fois de la francité et de quelque chose d'autre qui s'identifiera progressivement à l'américanité.

Volkswagen Blues semble remettre en question une identité nationale fondée exclusivement sur le relais français. Ce roman pose que le problème de l'identité québécoise ne saurait être résolu qu'en tenant compte simultanément de la francité et de l'américanité du Québécois. Son protagoniste est de toute évidence mécontent de lui-même et cherche à sortir de sa léthargie par l'intermédiaire d'un voyage fondamentalement géographique et historique à travers le continent nord-américain. Écrivain de profession et solitaire par surcroît, Jack Waterman vit exclusivement dans la francité,

4 Gilles Marcotte, « Découvrir l'Amérique ", Littérature et circonstances, Montréal, L'Hexagone, 1989, p. 91.

5 Jean Morisset, L'identité usurpée. L'Amérique écartée, Montréal, Éditions Nouvelle Optique, 1985.

6 Jacques Poulin, Les grandes marées, Montréal, Éditions Lernéec, 1978, p. 99. 
c'est-à-dire qu'en tant qu'écrivain québécois, il s'inscrit, bon gré mal gré, dans une culture véhiculée par la langue française qui lui sert d'outil. Or, la francité semble liée à la douceur, à l'immobilisme et à l'inertie généralement symptomatiques du scripteur poulinien. Les tendances introspectives de Noël, sa valorisation du passé (l'enfance), trahissent un protagoniste empêtré dans son repliement, dans son inactivité, comme dans la tendresse. Or l'apparente docilité de Noël, son manque de virilité et d'agressivité résultent de son opération (voir p. 154 et p. 185) mais ne lui sont pas spécifiques. L'identification de Noël au Vieux-Québec semble suggérer qu'il en est de même des Québécois en général. Or les caractéristiques spécifiques à Noël et par extension au Vieux-Québec sont imprégnées de connotations négatives une fois confrontées aux traits des héros de Noël. Dans les autres romans, les faiblesses du scripteur poulinien s'opposent le plus souvent aux qualités de son frère, Théo, personnification de l'américanité.

Mais avant d'entreprendre l'illustration de ce phénomène, il faut considérer une autre ramification de l'acception d'«Américain " proposée par Noël et repérable dans les autres romans. Je me réfère ici à l'idée d'agressivité et à son opposition à la docilité dans « l'espoir fou qu'on peut tout faire de force " et dans *il n'y a plus de place pour la douceur". Trait apparemment positif, la douceur, on le sait, est particulière aux protagonistes scripteurs de Poulin. Mais elle relève des aspects négatifs car elle est reliée, par le biais de l'écriture, à la dénégation de la vie. Ainsi, l'écriture dans Volkswagen Blues représente «une faf̧on de ne pas vivre» (p. 136). Dans Le coeur de la baleine bleue, la douceur amène la soumission, le rejet et finalement la mort.

Par contre, l'agressivité du joueur de hockey, Maurice Richard, est valorisée car elle éveille chez Noël comme chez d'autres « des émotions si profondes qu'elles touchaient aux racines les plus lointaines et jusqu'à ce fonds commun qui faisait notre racen (p. 92).

La figure de l'écrivain américain Ernest Hemingway fait ressortir l'inertie caractéristique du scripteur fictif poulinien. Dans Le ccur de la baleine bleue, Hemingway représente une passion pour la vie (p. 63) qui chez Poulin est associée de plus 
en plus à l'agressivité. Avec Le vieux chagrin, il devient clair qu'il faut entendre "agressivité " comme l'expression d'un * goût de vivre " ${ }^{7}$. Or cette force vitale de l'Hemingway poulinien sera incarnée à partir de Faites de beaux rêves par le frère, Théo. Dans Volkswagen Blues, celui-ci fait penser au coureur de bois Étienne Brûlé et, en général, aux pionniers nord-américains: «mon frère Théo, comme les pionniers, était absolument convaincu qu'il était capable de faire tout ce qu'il voulait * (p. 137). Théo se distingue donc du protagoniste scripteur (Amadou, Teddy, Jack Waterman) par son nomadisme, son agressivité et sa confiance en soi. Waterman compare les exploits de Théo, son courage, son dynamisme (p. 216-217), à sa propre mollesse et à sa timidité à l'égard de la vie (p. 211).

Isolé par l'écriture et bouleversé par un sentiment d'échec et d'anéantissement personnels, Waterman espère pouvoir trouver son salut chez son frère (p. 14, 123). La quête de Théo traduit une tentative pour se réconcilier avec autrui ou selon l'écrivain américain Saul Bellow: «When you're looking for your brother, you're looking for everybody !* (p. 110). La quête du frère est également la quête de soi car c'est par rapport à Théo que s'identifie Jack Waterman. Qui est donc Théo pour Jack Waterman? Un homme physiquement et moralement «le contraire de [lui]" (p. 13). Un champion et un voyageur insouciant: une sorte de coureur de bois à travers les souvenirs duquel Jack retrouve son enfance, une enfance et un bonheur indissociables de l'histoire de l'Amérique française et des ancêtres aventuriers. De cette héroïsation du frère résulte une figure légendaire, mythique (p. 217) à laquelle Jack Waterman voudrait pouvoir s'identifier (p. 109, 167) car il reconnaît en son frère cette * partie de [lui-même] qui a oublié de vivre" (p. 137). En quoi consiste la nature de Théo? Il me semble qu'elle se distingue par son nomadisme, par son esprit d'aventures et même par sa capacité de violence. Bref, par son américanité. Ainsi, les deux frères, Jack Waterman et Théo, incarnent corrélativement la sédentarité et le nomadisme constitutifs de la dualité du Québécois.

La quête de Théo vise le comblement d'un manque, de ces traits perdus ou refoulés chez le scripteur poulinien qui

7 Jacques Poulin, Le vieux chagrin, Montréal/Arles, Editions Leméac/Actes Sud, 1989, p. 139. 
s'associent, en particulier, au coureur de bois et au pionnier nord-américain. Waterman cherche cette partie de lui-même qui fut sacrifiée à la vie sédentaire imposée par l'écriture. Mécontent de lui-même, Jack Waterman semble vouloir récupérer son américanité en s'identifiant aux premiers Américains, à son frère et à ses ancêtres émigrés. La quête du frère franco-américain implique une reconnaissance intuitive de l'hybridité de l'ethnie québécoise. L'hybridité semble même être particulière à l'homme nord-américain. Et le coureur de bois est, par ses errances et son adoption de la vie indienne, un des premiers à en faire la preuve (p. 202). Le métissage est valorisé dans Volkswagen Blues (p. 224 et p. 259) et Waterman, comme Pitsémine (p. 81-82 et p. 224), doit réconcilier sa propre dualité. Son désir de retrouver en lui-même des traits de son frère aventurier se fonde principalement sur la reconnaissance de l'insuffisance et l'incomplétude de son identité personnelle. C'est en partant à la recherche de son frère aventurier que Jack Waterman explore son appartenance au continent nord-américain. La quête de Théo est en fin de compte la recherche d'un certain type d'homme, ou plutôt d'un certain état d'esprit, nécessaire à la réintégration de l'américanité. C'est une redécouverte de l'appartenance au continent américain et une nouvelle pénétration de l'Amérique par les Canadiens français qu'examine Volkswagen Blues. Et cette expansion identitaire se fait par le biais du voyage et de la redécouverte des traces de l'ancienne présence française (et indienne) en Amérique du Nord. Voici la façon dont Jacques Poulin considère la quête de Waterman et de Pitsémine: «Je crois qu'ils cherchent, sans trop s'en rendre compte, quelle est la place que la conscience française occupe en Ámérique, ou peut-être quelle est la part de l'âme québécoise qui est américaine. " ${ }^{8} \mathrm{Le}$ voyage marque la fin définitive de l'isolement, de l'aspect insulaire du Québec évoqué dans Les grandes marées et, en général, dans la fermeture spatiale des romans précédents.

Le coureur de bois est une figure clef dans Volkswagen Blues: il représente cette partie du Québécois en rupture de l'héritage français. La disparition de ce premier Américain au

8 Cité par Nicole Beaulieu dans « Jacques Poulin, l'écrivain dans l'ombre", L'actualité, vol. X, n 4, avril 1985, p. 77. 
profit de l'agriculteur mena au repliement sur soi, à l'immobilisme et inévitablement à la perte de l'américanité du Québécois. Ainsi le dualisme francité /américanité trouve donc une certaine équivalence dans la dichotomie sédentarité/nomadisme qui, quoique implicite chez Poulin, est très évidente dans le discours de certains critiques notamment chez Christian Morissonneau ${ }^{9}$, chez Jacques Languirand ${ }^{10}$ et chez Jean Morisset. Les deux composantes majeures de l'identité québécoise, la francité et l'américanité, s'associent généralement à deux types de colons français en Amérique: le sédentaire (ou l'habitant) et le nomade (ou le coureur de bois). D'après Michel Morin, l'idéologie nationaliste du Canada français lança un appel à la sédentarisation, à la colonisation des terres, à la fidélité aux origines françaises dans le but de contrecarrer deux tendances à la dispersion mettant en danger la survivance nationale. Il s'agit en premier lieu de *[l']appel de l'Indien, de la forêt, de l'exploration, du territoire continental » et ensuite, de «l'appel des États-Unis, de la démocratie à l'américaine, de la productivité liée aux chances d'ascension sociale " ${ }^{11}$. L'identification du Canadien français uniquement à la francité nécessita la répression de son américanité et donc de son hybridité.

Théo est associé non seulement au coureur de bois Étienne Brûlé mais aussi à Jack Kerouac (p. 74, p. 216), aventurier moderne et romancier franco-américain. Son roman, On the Road, intertexte important dans Volkswagen Blues, est un récit de voyage incitant tout lecteur à prendre la route dans une tentative de se réapproprier physiquement et spirituellement le continent nord-américain. L'esprit de $O n$ the Road, son génie pour l'aventure, le risque, le voyage, soutiennent la quête de Théo. L'élan qui pousse les protagonistes de Kerouac, Sal Paradise et Dean Moriarty, à travers l'Amérique ressemble à celui-là même qui incita les premiers pionniers à prendre la route: confiance en soi, désir de liberté, de bonheur et d'aventures. Mais Volkswagen Blues assimile

9 Voir La Terre promise: le mythe du Nord québécois, Montréal, Éditions Hurtubise HMH, 1978.

10 "Le Québec et l'Américanité ", Études littéraires, vol. VIII, n“ 1, avril 1975, p. 146.

11 Michel Morin, L'Amérique du Nord et la culture. Le territoire imaginaire de la culture. Tome II, Ville La Salle, Éditions Hurtubise HMH, 1982, p. 124. 
moins l'aspect *Beat* de On the Road que sa remise en question de l'Amérique et du rêve américain.

Alors qu'en est-il de l'Amérique et du rêve américain dans le roman de Poulin? À première vue, l'Amérique dans Volkswagen Blues évoque la liberté, le bonheur, la possibilité de tout oublier et de recommencer à neuf dans un nouveau monde. Le mot *Amérique * résonne même aujourd'hui de ces associations.

L'Amérique! Chaque fois qu'il entendait prononcer ce mot, Jack sentait bouger quelque chose au milieu des brumes qui obscurcissaient son cerveau. (Un bateau larguait ses amarres et quittait lentement la terre ferme.) C'était une idée enveloppée de souvenirs très anciens - une idée qu'il appelait le * Grand Rêve de l'Amérique ". II pensait que, dans I'histoire de l'humanité, la découverte de l'Amérique avait été la réalisation d'un vieux rêve. Les historiens disaient que les découvreurs cherchaient des épices, de l'or, un passage vers la Chine, mais Jack n'en croyait rien. Il prétendait que, depuis le commencement du monde, les gens étaient malheureux parce qu'ils n'arrivaient pas à retrouver le paradis terrestre. Ils avaient gardé dans leur tête l'image d'un pays idéal et ils le cherchaient partout. Et lorsqu'ils avaient trouvé l'Amérique, pour eux c'était le vieux rêve qui se réalisait et ils allaient être libres et heureux. Ils allaient éviter les erreurs du passé. Ils allaient tout recommencer à neuf. (p.100-101)

Ce passage révèle que le concept de * grand rêve américain *, comme le but du voyage que fit Jacques Cartier en Amérique ${ }^{12}$, est double et comprend un trait distinctif matériel (épices, or) et un autre spirituel (paradis terrestre, liberté, bonheur). C'est à ce dernier aspect que s'intéresse l'écrivain Jack Waterman. Mais l'Amérique, dans Volkswagen Blues, est essentiellement un rêve qui s'évanouira, un mythe qui se désintégrera. Il est donc normal que Jack Waterman trouve la carte postale évoquant la découverte de l'Amérique par Jacques Cartier (p. 19) dans un livre intitule The Golden Dream et que c'est là qu'il puise également la légende de l'Eldorado.

12 Voir La découverte ambiguē d'André Berthiaume, Montréal, Le Cercle du Livre de France, 1976. 
Or, le thème de l'Amérique soulève en lui-même une problématique chère à Jacques Poulin: celle des rapports humains. C'est ce qui intéresse également Tzvetan Todorov dans La conquête de l'Amérique. La question de l'autre (Seuil, 1982). Dans les deux textes, il est clair que l'Amérique fut peuplée à partir de deux objectifs diamétralement opposés: l'un spirituel ou religieux (ou plus tard démocratique), l'autre matériel ou économique. C'est ce que révèle également le récit de Jacques Cartier imprimé sur la carte postale envoyée par Théo. Les romans de Saul Bellow et de Jack Kerouac représentés dans Volkswagen Blues aussi mettent en scène ces deux aspects contradictoires de l'Amérique et du rêve américain. Comme Volkswagen Blues, ces intertextes privilégient l'aspect spirituel de l'Amérique et du rêve américain, c'est-àdire la problématique du bonheur, de la quête de soi et de l'harmonie entre les hommes, bref la régénération de l'homme en Amérique.

Mais dès le commencement du voyage, l'allusion à The Hotel New Hampshire de John Irving place la quête de Jack Waterman et des pionniers sous le signe de l'échec: le rêve américain est un leurre. La découverte de l'Amérique devait servir à l'expansion du christianisme et donc au bien-être spirituel de l'homme nord-américain. Mais le tout premier contact de l'Européen avec l'indigène fut un contact violent fondé sur l'abaissement et l'anéantissement de celui-ci dans la tentative de l'assimiler, de s'élever en maître et de s'enrichir. Ainsi les nombreux récits faits par la Métisse sur l'extermination des Indiens révèlent que les facettes matérielle et spirituelle de l'Amérique et du rêve américain sont irréconciliables: la quête de richesses et l'éclatement de la violence sont intrinsèques à l'établissement de l'Amérique (p.128, 208). Que l'on parle des assassinats des Indiens par Cavelier de La Salle, du massacre des pionniers, ou de Chicago, la ville d'Al Capone et d'Eliot Ness (p.103), « on commence à lire l'histoire de l'Amérique et il y a de la violence partout. On dirait que toute l'Amérique a été construite sur la violence» (p. 129).

Les récits historiques de Pitsémine s'opposent donc aux récits d'enfance et d'innocence que raconte Jack Waterman, toujours aveuglé par les exploits des aventuriers de la Nouvelle France. Les récits de Pitsémine signalent la 
dégradation des rapports humains dans le Nouveau Monde, la corruption de la Terre Promise et donc l'échec du rêve américain spirituel: "Avec le temps, le "Grand Rêve de l'Amérique " s'était brisé en miettes comme tous les rêves " (p. 101).

Volkswagen Blues exprime ainsi une certaine déception à l'égard de l'Amérique et du rêve américain; la dégradation de l'image de Théo est étroitement liée à la désintégration de l'image de l'Amérique. La caractérisation progressivement négative de Théo ${ }^{13}$, à l'instar du coureur de bois Étienne Brûlé, s'accorde avec l'image de traître qui lui est imposée à la fin du roman (p. 267) et avec la trahison des espoirs humains par l'Amérique. Le voyage de Gaspé à San Francisco permet à Waterman de prendre conscience de son appartenance à l'Amérique mais il lui dévoile aussi l'impuissance de celle-ci à répondre aux rêves et aux espoirs de l'humanité. L'histoire de la terre américaine est une histoire sanglante qui fait un leurre du grand rêve américain: la liberté, le bonheur, l'harmonie entre les hommes ne sont pas plus possibles en Amérique qu'ailleurs. Les nouvelles à la radio annoncent la violence et l'absence de bonheur en Amérique et dans le monde entier (p. 100). Et seul le commerce règne là où le mouvement des hippies avait essayé « de mettre en pratique [...] une nouvelle conception de la vie et des rapports entre les gens " (p. 278).

Pour ce «doux * timide qu'est Jack Waterman, l'américanité est attirante mais en même temps répugnante par son aspect immoral et violent. Le retour chez soi, le retour au Québec semble être la meilleure solution pour Jack Waterman. A la fin de son voyage à travers l'Amérique, il trouve son frère Théo, ce personnage devenu pour lui mythique, presque légendaire, tel qu'il est véritablement: un homme pitoyable qui a subi les ravages du temps et d'une vie mouvementée, un homme beat tels Dean Moriarty à la fin de On the Road et Jack Kerouac lui-même à la fin de sa vie. L'évocation de Kerouac, dans Volkswagen Blues, est centrale

13 Jack Waterman et Pitsémine découvrent que Théo fut arrété à Toronto pour la possession d'une arme à feu, qu'il fut détenu comme suspect pour le vol au Kansas City Museum (p. 139) et que ce vol provoqua la mort du gardien (p. 190). 
à toute la problématique de la quête d'identité. Elle attire l'attention sur l'aventurier moderne, sur un type d'homme dont l'identité est ancrée dans le tellurisme nord-américain. Mais la déchéance de Kerouac, comme de Théo, est représentative du sort du Franco-Américain, de sa difficulté à assumer entièrement l'Amérique et de son impossibilité à intégrer un rêve américain foncièrement égoïste et matérialiste.

Le coureur de bois, l'aventurier qui attire Jack Waterman ne se retrouve plus chez Théo paralytique amnésique. Théo n'est pas en mesure de répondre aux besoins personnels de Jack Waterman, tout comme celui-ci n'a pas su répondre à ceux de Théo des années précédentes (p. 78). La recherche de Théo le pousse à faire l'exploration géographique et historique du continent américain mais sa déchéance, la perte de sa mémoire et de sa langue ancestrale comme de son identité (p. 266, p. 288) font que Jack Waterman ne saurait ni embrasser l'exil, ni assumer entièrement son américanité: la proximité géographique des États-Unis implique, en fin de compte, son américanisation.

Il me semble que la condition lamentable de l'exilé Théo à la fin du roman est significative. Le destin tragique de Théo, son rapprochement de Jack Kerouac, la valorisation du Nord par rapport au Sud et du sédentaire (les colons de l'Oregon) par rapport au nomade (l'aventurier en quête d'or de la Californie) trahissent un certain penchant conservateur présent dans Volkswagen Blues et cela en dépit de son apparente ouverture, du moins spatiale et historique, à l'égard de l'Amérique. L'Amérique chez Poulin est essentiellement un mythe et rien ne garantit à l'exilé québécois la réalisation de son rêve américain. 